\title{
Expansion of vdelta1 T lymphocytes reactive to c. albicans IN HIV-1 infected patients: effect of influenza virus vaccine
}

\author{
Maria Raffaella Zocchi ${ }^{1 *}$, Daniela Fenoglio ${ }^{2}$, Alessia Parodi $^{2}$, Alessandra Ferrera $^{2}$, Paolo Durando $^{3}$, \\ Roberto Gasperini ${ }^{3}$, Silvia Catellani ${ }^{4}$, Alessandro Poggi $i^{5}$ \\ From $16^{\text {th }}$ International Symposium on HIV and Emerging Infectious Diseases \\ Marseille, France. 24-26 March 2010
}

\section{Background}

It is known that the circulating Vdelta2 $\mathrm{T}$ cell subset respond to mycobacteria and certain viruses, while the Vdelta1 subset is resident in the mucosal-asssociated lymphoid tissue and participate in the immunity against intracellular microrganisms. We reported that in HIV-1 infected patients circulating Vdelta1 $\mathrm{T}$ lymphocytes are increased; in vitro, these cells can proliferate in response to Candida albicans. We analysed the effects of influenza virus vaccination on the function of this $\mathrm{T}$ cell subset in HIV-1 infected patients and healthy donors.

\section{Methods}

We analysed the effects of influenza virus vaccination on the function of Vdelta1 and Vdelta2 $\mathrm{T}$ cell subsets in HIV-1 infected patients and healthy donors. Cells were isolated from blood samples obtained before and after 30 or 90 days after vaccination. Proliferation to C.albicans and to hemoagglutimnin (HA) was assessed by thymidine uptake after 7 days of stimulation.

\section{Results}

First, we confirmed that the Vdelta1 $\mathrm{T}$ cell subset is expanded in HIV-1 infected patients (absolute number of cells/microliter range 28-30 in HIV-1 patients vs. 812 in healthy donors). On day 90 after vaccination the number of Vdelta1 $\mathrm{T}$ cells significantly increased in HIV-1 patients (59 in the group A, 48 in the group B). Interestingly, upon influenza vaccination an increase in proliferation of Vdelta1 $\mathrm{T}$ cells to $\mathrm{C}$. albicans was observed in HIV-1 patients, at variance with healthy donors, on day 30 and day 90. A specific cellular response to HA was detectable in HIV-1 patients only on day 90 post-vaccination without MF59 adjuvant, but it was observed on day 30 , as in seronegative subjects, when MF59-vaccine was used.

\section{Discussion}

We suggest that in HIV-1 infected patients, a population of Vdelta1 $\mathrm{T}$ lymphocytes reactive to $\mathrm{C}$. albicans is present in vivo; upon challenge with influenza virus vaccine this population receives an activation signal possibly mediated by cytokines triggered by the $\mathrm{HA}$ antigen itself.

\section{Author details}

IScientific Institute San Raffaele, Department of Immunology, Milan, Italy. ${ }^{2}$ CEBR, University of Genoa, Genoa, Italy. ${ }^{3}$ Department of Health Science, San Martino Hospital, Genoa, Italy. ${ }^{4}$ Department of Oncohematology, Universiti of Genoa, Genoa, Italy. ${ }^{5}$ National Institute for Cancer Research, Unit of Molecular Oncology and Angiogenesis, Genoa, Italy.

Published: 11 May 2010

\section{doi:10.1186/1742-4690-7-S1-P25}

Cite this article as: Zocchi et al:: Expansion of vdelta1 $\mathrm{T}$ lymphocytes reactive to $c$. albicans IN HIV-1 infected patients: effect of influenza virus vaccine. Retrovirology 2010 7(Suppl 1):P25.

* Correspondence: zocchi.maria@hsr.it

${ }^{1}$ Scientific Institute San Raffaele, Department of Immunology, Milan, Italy 